\title{
Theoretical studies of possible toroidal high-spin isomers in the light-mass region
}

\author{
Andrzej Staszczak ${ }^{1}$, Cheuk-Yin Wong ${ }^{2}$
}

${ }^{1}$ Institute of Physics, Maria Curie-Skłodowska University, Lublin, Poland

${ }^{2}$ Physics Division, Oak Ridge National Laboratory, Oak Ridge, TN USA

\begin{abstract}
We review our theoretical knowledge of possible toroidal high-spin isomers in the light mass region in $28 \leq A \leq 52$ obtained previously in cranked Skyrme-Hartree-Fock calculations. We report additional toroidal high-spin isomers in ${ }^{56} \mathrm{Ni}$ with $I=114 \hbar$ and $140 \hbar$, which follow the same (multi-particle)-(multi-hole) systematics as other toroidal high-spin isomers. We examine the production of these exotic nuclei by fusion of various projectiles on ${ }^{20} \mathrm{Ne}$ or ${ }^{28} \mathrm{Si}$ as an active target in time-projection-chamber (TPC) experiments.
\end{abstract}

\section{Introduction}

Wheeler suggested that under appropriate conditions the nuclear fluid may assume a toroidal shape [1]. If toroidal nuclei could be made, there would be a new family tree for the investigation of the nuclear species.

The rotating liquid-drop model is useful as a qualitative guide to point out essential energy balances leading to possible toroidal figures of equilibrium [2]. A quantitative assessment relies on microscopic descriptions that include both the bulk properties of the nucleus and the single-particle shell effects in self-consistent mean-field theories, such as the Skyrme-HartreeFock (SHF) approach [3]. The non-collective rotation with an angular momentum about the symmetry axis is permissible quantum mechanically for an axially symmetric toroid by making particle-hole excitations and aligning the angular momenta of the constituents along the symmetry axis [4]. As 
a consequence, only a discrete, quantized set of total angular momentum $I=I_{z}$ states is allowed.

In our recent works [5], we showed by using a cranked SHF approach that toroidal high-spin isomeric states have general occurrences in $28 \leq A \leq 52$ for even-even $N=Z$ and $N \neq Z$ nuclei. Toroidal high-spin isomers have also been found theoretically in similar HF calculations in this mass region [6].

We would like to review the systematics of these nuclei and suggest ways how these nuclei may be produced.

\section{Light-mass toroidal high-spin isomers}

We have located the toroidal high-spin isomers at their energy minima using the cranked SHF approach [5]. We find that the ratio of the torus major radius $R$ to the torus minor radius $d, R / d$, increases with angular momentum and approximately linearly with the mass number while the minor radius $d$ remains essentially unchanged (see Ref. [5]). It is useful to classify these nuclei according to the $n$-particle $n$-hole nature of the isomer, relative to the toroidal nucleus at $I=0$. One finds that all $n \mathrm{p}-n \mathrm{~h}$ families follow a regular well-behaved pattern as shown in Fig. 1, where we plot the total energy $E^{\text {tot }}(I)$ of the isomer ${ }^{A} Z^{t}(I)$ as a function of $R / d$ for different toroidal isomers with various aligned angular momenta $I$.

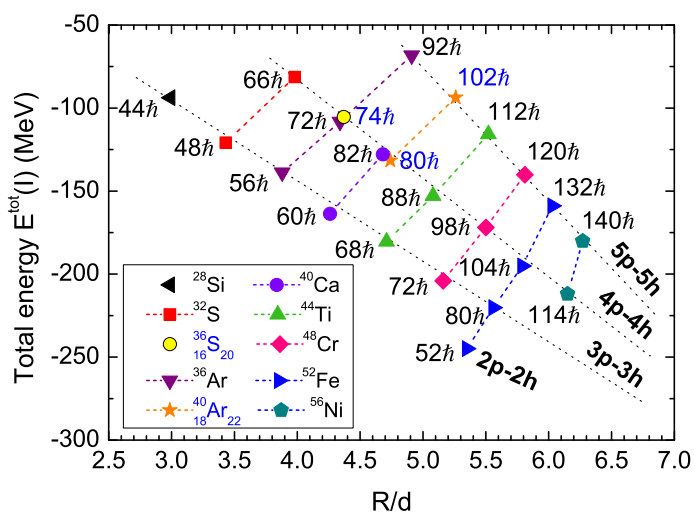

Figure 1: (Color online.) The total energies of the isomeric toroidal states of ${ }_{14}^{28} \mathrm{Si}$, ${ }_{16}^{32} \mathrm{~S},{ }_{16}^{36} \mathrm{~S},{ }_{18}^{36} \mathrm{Ar},{ }_{18}^{40} \mathrm{Ar},{ }_{20}^{40} \mathrm{Ca},{ }_{22}^{44} \mathrm{Ti},{ }_{24}^{48} \mathrm{Cr},{ }_{26}^{52} \mathrm{Fe}$, and ${ }_{28}^{56} \mathrm{Ni}$ and their associated angular momenta $I=I_{z}$ values along the symmetry axis, as a function of $R / d$. The $n \mathrm{p}-n \mathrm{~h}$ configurations relative to the $I=0$ states are also indicated.

We collect the properties of all known 21 toroidal high-spin isomers up to ${ }^{52} \mathrm{Fe}$ obtained previously in [5] in Fig. 1 . These systematics predict the 
possible presence of $n \mathrm{p}-n \mathrm{~h}$ toroidal high-spin isomers for ${ }^{56} \mathrm{Ni}$ at $R / d \sim$ 6.0. Indeed, we found energy minima for ${ }^{56} \mathrm{Ni}$ with $I=114 \hbar$ and $140 \hbar$ at $R / d=6.15$ and 6.27 , respectively, in subsequent cranked SHF calculations.

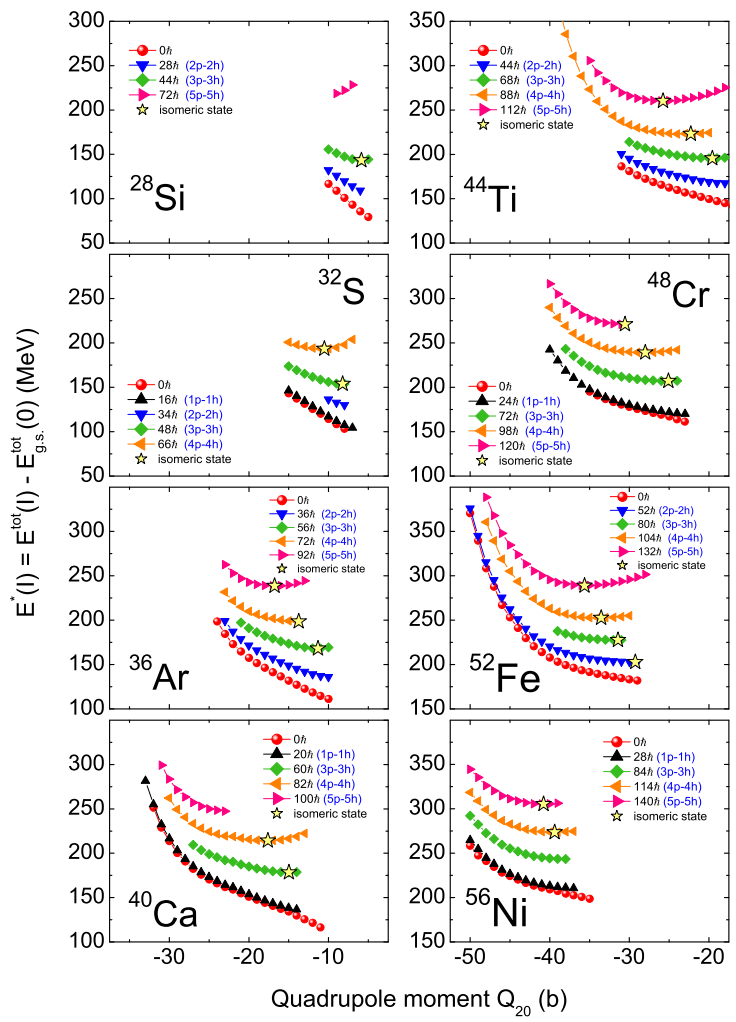

Figure 2: (Color online.) The excitation energies $E^{*}(I)$ of high-spin toroidal states of ${ }^{28} \mathrm{Si},{ }^{32} \mathrm{~S},{ }^{36} \mathrm{Ar},{ }^{40} \mathrm{Ca},{ }^{44} \mathrm{Ti},{ }^{48} \mathrm{Cr},{ }^{52} \mathrm{Fe}$, and ${ }^{56} \mathrm{Ni}$ as a function of the quadrupole moment $Q_{20}$ for different angular momenta along the symmetry axis, $I=I_{z}$.

In Fig. 2 the excitation energies $E^{*}(I)$ of even-even $N=Z$ toroidal states relative to the energy of the ground states are presented as a function of the quadrupole moment $Q_{20}$ for different $I$ values along the symmetry axis. They include those from our earlier work in [5] and the newly found ${ }^{56} \mathrm{Ni}$ toroidal isomers. The locations of energy minima $E^{*}\left({ }^{A} Z^{t}(I)\right)$ representing toroidal high-spin isomeric states are indicated by star symbols.

\section{Production of light-mass toroidal isomers}

As the question of the mean half-life of these isomers remains unresolved, one can design experiments that could detect the existence of the exotic toroidal 
high-spin nuclei of Figs. 1 and 2 at appropriate energies, if the mean halflives are longer than $\hbar / \mathrm{MeV}$ or $200 \mathrm{fm} / \mathrm{c}$. One way is to search for these isomers as resonances or metastable nuclei by bombarding projectile nucleus ${ }^{A_{p}} Z_{p}$ on an active-target nucleus ${ }^{A_{T}} Z_{T}$ for the production of the toroidal high-spin isomer ${ }^{A} Z^{t}(I)$ with angular momentum $I=I_{z}$,

$$
{ }^{A_{p}} Z_{p}+{ }^{A_{T}} Z_{T} \rightarrow{ }^{A} Z^{t}(I) .
$$

The active target can be, for example, ${ }^{20} \mathrm{Ne},{ }^{36,38,40} \mathrm{Ar}$, or ${ }^{28} \mathrm{Si}$. We shall consider the cases of ${ }^{20} \mathrm{Ne}$ and ${ }^{28} \mathrm{Si}$ as active-targets.

In recent years, TPC chambers have been used to study the nuclear spectroscopy of metastable nuclei $[7,8]$. The idea is to use a chamber of noble gas under a high voltage so that the gas itself or an embedded solid layer serves as the target, and the nuclear trajectories show up as tracks. The production of a composite nucleus with a long half-life would show up as a single track with the mass and charge arising from the fusion of the projectile and target nuclei. The production of binary products indicates a two $\rightarrow$ two reaction from which one can examine the elastic and inelastic channels and study the excitation function and angular distribution to search for various meta-stable states. Previously, many metastable states formed by colliding various projectile nuclei with an active He target have been found by such a technique [7].

The cross section for producing a toroidal isomer at the correct energy and angular momentum is [9] (p. 517)

$$
\sigma_{\text {res }}\left(E,{ }^{A} Z^{t}(I)\right)=\frac{4 \pi}{k^{2}}(2 I+1) \frac{\Gamma^{2} / 4}{\left[E-E_{\text {res }}\left({ }^{A} Z^{t}(I)\right)\right]^{2}+\Gamma^{2} / 4} B_{\text {in }} B_{\text {out }},
$$

where $E$ is the c.m. energy, $E_{\text {res }}\left({ }^{A} Z^{t}(I)\right)$ is the c.m. resonance energy for the toroidal high-spin isomer with spin $I, k$ is the c.m. momentum in the initial state, and $\Gamma$ is the full width at half maximum height of the resonance. The quantities $B_{\text {in }}$ and $B_{\text {out }}$ are the branching fractions for the resonance into the initial-state and finial-state channel, respectively. Here, the width $\Gamma$ and the branching fractions $B_{\text {in }}$ and $B_{\text {out }}$ may need to be determined experimentally. The resonance energy $E_{\text {res }}$ (in the c.m. system) is given by

$$
E_{\mathrm{res}}\left({ }^{A} Z^{t}(I)\right)=M\left({ }^{A} Z^{t}(I)\right)-M\left({ }^{A_{T}} Z_{T}\right)-M\left({ }^{A_{p}} Z_{p}\right),
$$

where $M\left({ }^{A} Z^{t}(I)\right), M\left({ }^{A_{T}} Z_{T}\right)$, and $M\left({ }^{A_{p}} Z_{p}\right)$ are nuclear masses of ${ }^{A} Z^{t}(I)$, $A_{T} Z_{T}$, and ${ }^{A_{p}} Z_{p}$, respectively. In terms of the binding energies $B\left({ }^{A} Z\right)$, $B\left({ }^{A_{T}} Z_{T}\right)$, and $B\left({ }^{A_{p}} Z_{p}\right)$, and excitation energy $E^{*}\left({ }^{A} Z^{t}(I)\right)$, we have

$$
E_{\mathrm{res}}\left({ }^{A} Z^{t}(I)\right)=E^{*}\left({ }^{A} Z^{t}(I)\right)-B\left({ }^{A} Z\right)+B\left({ }^{A_{T}} Z_{T}\right)+B\left({ }^{A_{p}} Z_{p}\right) .
$$



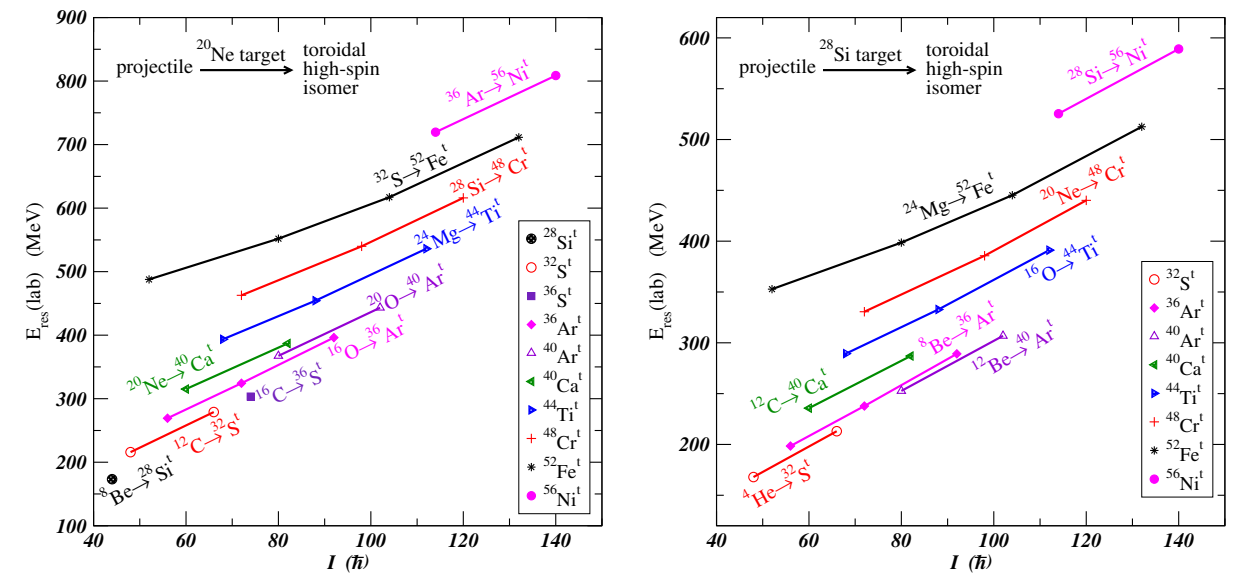

Figure 3: (Color online) The resonance energies $E_{\text {res }}\left({ }^{A} Z^{t}(I)\right)$ (lab) in the laboratory system in the bombardment of various projectiles ${ }^{A_{p}} Z_{p}$ on ${ }^{20} \mathrm{Ne}$ (left panel) or ${ }^{28} \mathrm{Si}$ (right panel) as an active target for the production of toroidal isomers ${ }^{A} Z^{t}(I)$ with different angular momenta $I$.

The resonance energy $E_{\mathrm{res}}\left({ }^{A} Z^{t}(I)\right)$ (lab) in the laboratory system is given by $E_{\text {res }}\left({ }^{A} Z^{t}(I)\right) \times\left(A_{p}+A_{T}\right) / A_{T}$. For the production of toroidal highspin isomers by collision on ${ }^{20} \mathrm{Ne}$ or ${ }^{28} \mathrm{Si}$ as an active target, the resonance energies are shown in Fig. 3. The knowledge of the predicted values of $E_{\text {res }}\left({ }^{A} Z^{t}(I)\right)(\mathrm{lab})$ will facilitate the search of toroidal high-spin isomers.

\section{Conclusions and discussion}

Under (multi-particle)-(multi-hole) excitation involving large orbital angular momentum orbitals, non-collective rotations of many light nuclei lead to equilibrium configurations whose densities may assume the shape of a torus. The $n \mathrm{p}-n \mathrm{~h}$ systematics of toroidal high-spin isomers fit a regular pattern which can be used to predict possible presence of toroidal high-spin isomer in ${ }^{56} \mathrm{Ni}$. We found additional equilibrium energy minima for ${ }^{56} \mathrm{Ni}$ with $I=114 \hbar$ and $140 \hbar$ in subsequent cranked SHF calculations.

We examine the production of light-mass toroidal high-spin isomers by fusion of various projectile nuclei with an active target, in which the trajectories of the reaction products can be examined as a function of the collision energies. Resonance energies for the production of toroidal high-spin isomers have been calculated for ${ }^{20} \mathrm{Ne}$ or ${ }^{28} \mathrm{Si}$ as an active target, based on the Skyrme-Hartree-Fock energies obtained for the isomers.

The technology of building a TPC using $90 \%$ of ${ }^{20} \mathrm{Ne}$ as its dominant ingredient has been developed by the ALICE collaboration [10]. The uti- 
lization of a similar TPC detector with ${ }^{20} \mathrm{Ne}$ or ${ }^{28} \mathrm{Si}$ as an active target for nuclear spectroscopy may prove useful for the search of toroidal isomers.

\section{Acknowledgements}

The authors wish to thank Profs. J. Natowitz and K. Read for helpful discussions. This work was supported in part by the Division of Nuclear Physics, U.S. Department of Energy, Contract No. DE-AC05-00OR22725.

\section{References}

[1] See a reference to J. A. Wheeler's toroidal nucleus in Gamow G. 1961 Biography of Physics (New York: Harper \& Brothers Publishers) pp. 297.

[2] Wong C.Y., Ann. Phys. 77 (1973) 279; Phys. Rev. C 17 (1978) 331.

[3] Vautherin D. and Brink D.M., Phys. Rev. C 5 (1972) 626; Engel Y.M., Brink D.M., Goeke K., Krieger S., and Vautherin D., Nucl. Phys. A 249 (1975) 215.

[4] Bohr A. and Mottelson B.R., Nucl. Phys. A 354 (1981) 303c.

[5] Staszczak A. and Wong C.Y., Phys. Lett. B 738 (2014) 401; Acta Phys. Pol. B 46 (2015) 675, arXiv:1504.07646; Phys. Scripta (2015) in press, arXiv:1412.0050.

[6] Ichikawa T., Maruhn J.A., Itagaki N., Matsuyanagi K., Reinhard P.G., Ohkubo S., Phys. Rev. Lett. 109 (2012) 232503; Ichikawa T., Matsuyanagi K., Maruhn J.A., and Itagaki N., Phys. Rev. C 89 (2014) 011305(R); Phys. Rev. C 90 (2014) 034314.

[7] Suzuki D. et al., Nucl. Instr. Meth. A 691 (2012) 39; Suzuki D. et al., Phys. Rev. C 87 (2013) 054301.

[8] Roger T. et al., Nucl. Instr. Meth. A 638 (2011) 134, arXiv:1012.3560.

[9] Olive K.A. et al., (Particle Data Group), Chin. Phys. C 38 (2014) 1.

[10] B. Ketzer for the GEM-TPC, ALICE TPC Collaborations, Nucl. Instr. Meth. A 732 (2013) 237, arxiv:1303.6694. 